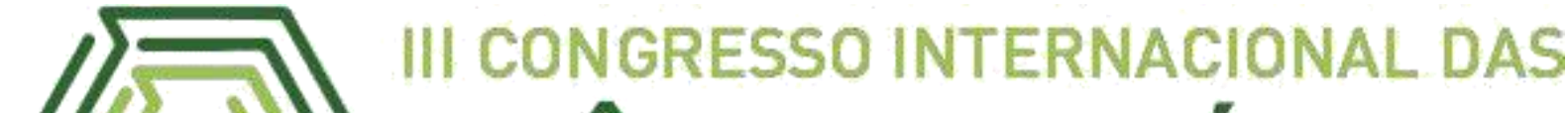 CIÊNCIAS AGRÁRIAS COINTER - PDVAGRO 2018:
}

\section{DENSIDADE POPULACIONAL DE MOSCA BRANCA Bemisia tabaci MEAN1 EM CULTIVARES DE SOJA $B t$}

\section{POPULATIONAL DENSITY OF THE SILVERLEAF WHITEFLY, Bemisia tabaci MEAN1, ON Bt SOYBEAN CULTIVARS}

\author{
Apresentação: Pôster
}

Luana Fernandes Barros ${ }^{1}$; Júlio Oliveira Anastácio ${ }^{2}$; Edson Araújo de Amorim ${ }^{3}$; Mauricélia Ferreira Almeida ${ }^{4}$; Edmar de Souza Tuelher ${ }^{5}$

\section{DOI: https://doi.org/10.31692/2526-7701.IIICOINTERPDVAGRO.2018.00412}

\section{Introdução}

A soja (Glycine Max L.) é uma das principais culturas agrícolas no Brasil e no mundo, tendo um importante valor socioeconômico, sendo utilizada tanto na alimentação humana animal, assim como é matéria prima em diversos complexos agroindustriais (MAUAD et al., 2010). O Brasil é o segundo maior produtor mundial deste grão, com uma produção estimada de 117 milhões de toneladas na safra 2017/2018. (CONAB, 2018).

Porém, a cultura da soja ainda apresenta sérios problemas com ataque de pragas, principalmente lagartas desfolhadoras que são pragas chaves desta cultura (MOSCARDI et al., 2012).Com a finalidade de reduzir as perdas causadas por estes insetos tem sido amplamente utilizado cultivares de soja geneticamente modificadas (GM) resistentes a estas lagartas que expressam proteínas inseticidas de Bacillus thuringiensis (Bt)(JAMES, 2015). Entretanto, pouco se sabe a respeito do efeito indireto destas proteínas sobre insetos não alvos, como a mosca branca BemisiatabaciMEAN1, principalmente devido à diminuição da aplicação de inseticidas para o controle das lagartas desfolhadoras.

Assim devido ao aumento da incidênciade B. tabaci em cultivos de soja, objetivou-se no presente estudoquantificar a densidade populacional assim como determinar a flutuação populacional de B. tabaci em cultivares de soja convencional e GM resistente a

\footnotetext{
${ }^{1}$ Engenharia Agronômica, Universidade Estadual da Região Tocantina do Maranhão, luananandes9@gmail.com

${ }^{2}$ Engenharia Agronômica, Universidade Estadual da Região Tocantina do Maranhão, aanastaciojulio07@gmail.com

${ }^{3}$ Engenharia Agronômica, Universidade Estadual da Região Tocantina do

Maranhão, edsonamorins@hotmail.com
} 
${ }^{4}$ DS em Entomologia, Universidade Estadual da Região Tocantina do Maranhão, mauricellia1@hotmail.com ${ }^{5}$ DS em Entomologia, Universidade Estadual da Região Tocantina do Maranhão, edmar.tuelher@gmail.com 
Lagartas desfolhadoras.

\section{Fundamentação Teórica}

A cultura da soja ainda possui sérios problemas ligados ao ataque de pragas durante todo seu ciclo, resultando em reduções na produção que podem chegar a $15,6 \%$ do total produzido (FAO, 2013). Apesar da ampla utilização da soja Bt visando reduzir o ataque de lagartas, o impacto desta tecnologia sobre população dos insetos sugadores ainda não foi completamente elucidado (OLIVEIRA et al., 2017).

Diante da utilização de cultivares resistentes ao ataque das principais lagartas desfolhadoras (e.g., Anticarsia gemmatallis e Chrysodeixis includens) ocorre a diminuição na aplicação de inseticidas para o controle destas pragas, podendo haver então o aumento na população de insetos não alvos como, por exemplo, a mosca branca, B. tabaci. Este inseto praga causa danos diretos através da sucção da seiva da planta, ou indiretos através da inoculação de toxinas e a transmissão do vírus "necrose-da-haste" (SILVA et al., 2009). Além disso, a excreção do inseto, conhecida como "honeydew", é rica em açúcares e serve como substrato para o estabelecimento do fungo que causa a fumagina (Capnodiums pp., Capnodiaceae) afetando principalmente a fotossíntese (LOPEZ et al., 2008).

Dessa forma, cultivares com menor potencial de serem bons hospedeiros para $B$. tabaci seriam mais rentáveis devido à menor incidência do inseto e, consequentemente,menores perdas devido ao ataque deste inseto praga.

\section{Metodologia}

O delineamento utilizado foi de blocos ao acaso, com três tratamentos e cinco repetições. Cada unidade experimental foi constituída por 6 linhas de 5 metros com espaçamento de $0,45 \mathrm{~m}$ entre linhas. A parcela útil foi constituída das duas linhas centrais, descartando-se $50 \mathrm{~cm}$ de cada lado da parcela, tendo, portanto,uma área útil de $3,6 \mathrm{~m}^{2}$ por parcela. Na bordadura da área experimental foi plantada a cultivar não Bt, BRS Sambaíba RR.

Foramtestadas três cultivares de soja comumente utilizadas nas regiões produtoras do Maranhão: a cultivar não Bt99R09 (Pioneer Sementes S.A) e duas cultivares de soja BtM8372 IPRO e a M8349 IPRO (Monsanto do Brasil S.A.). Foram realizados todos os tratos culturais necessáriosdurante o ciclo da cultura e utilizada irrigação por microaspersãoa cada dois dias.

Para determinação da incidência e flutuação populacional de B. tabaci foram 
realizadas amostragens semanais na parte da manhã, com a ocorrência da primeira avaliação 30 dias após o plantio a última avaliação quando da ocorrência da senescência das folhas de soja. A incidência dos adultos de B. tabaci foi determinada por meio de contagem direta do número de adultos do inseto em um folíolo do terço superior por planta, totalizando dez folíolos por parcela. Para a determinação da incidência de ovos e ninfas de B. tabaci foi retirado um trifólio no terço superior de cada planta totalizando dez plantas por parcela. Após a coleta, as folhas foram levadas para o laboratório de Entomologia da Universidade Estadual da Região Tocantina do Maranhão (UEMASUL). O número de ovos e ninfas de mosca branca foram contabilizados na face abaxial dos folíolos coletadoscom o auxílio de um microscópio estereoscópio.O número de ovos, ninfas e adultos de $B$. tabaci foram submetidos à análise de variância por medidas repetidas usando-se o procedimento PROC GLM (SAS INSTITUTE, 1989).

\section{Resultados e Discussões}

Foi observado diferença significativa entre as cultivares avaliadas para a densidade de adultos de B. Tabaci e para o número total de insetos,somando-se o número total de indivíduos de todos estádios de B. tabaci (Tabela 1). Houve diferença significativa com relação à flutuação populacional na densidadede adultos, sendo que na primeira avaliação houve menor incidência na cultivar Bt M8372 IPRO se comparada às demais (cultivar não Bt99R09 e cultivar BtM8349 IPRO)(Fig. 1A). Com relação ao número total de indivíduos, houve menor densidade para a cultivar BtM8372 IPRO apenas na terceira avaliação, naõ havendo diferença nas demais datas (Fig. 1B). Para os demais estádios de desenvolvimento não foi observada diferença na flutuação populacional entre os cultivares (Tabela 1, Fig. 1C-D).

Sendo assim, não Foi observada diferença na densidade e na flutuação populacional de mosca branca durante o período avaliado entre as cultivares Bt e não Bt estudadas. similarmente ao encontrado por Oliveira et al. (2018) que ao avaliar durante a safra 2015/2016 a dinâmica populacional de Bemisia tabaci em cultivares de soja transgênica concluíram que não houve impacto do uso da tecnologia Bt sobre B. tabaci.

A maior densidade de mosca branca, independente do cultivar e do estádio de desenvolvimento do inseto, ocorreu no início da fase reprodutiva da cultura da soja, decrescendo nas avaliações seguintes e mantendo-se baixo até o final do ciclo da cultura. Dessa forma, as variáveis ambientais parecem ter sido os principais fatores a 
influenciar a incidência da mosca branca,como pode ser verificada pela variação ao longo do tempo da mosca branca, sem haver diferença significativa entre os cultivares. Adicionalmente, as características da planta hospedeirapode também ter influenciado a população da mosca branca à medida em que mudanças da fenologia da planta com o avançar da fase reprodutiva (por exemplo a morfologia, idade e a coloração da folhas) podem afetar o desenvolvimento dos insetos ou até mesmo a taxa de oviposição dos insetos (CHU et al. 2001). Em nosso estudo, a maior incidência de $B$. tabaci encontrado no início do ciclo da cultura e no final da fase vegetativa e início da fase reprodutiva da cultura soja, coincidiram com a presença de vegetação mais tenra e folhas jovens mais jovens.

Assim, entre os cultivares testados, não se espera que ocorre um efeito benéfico ou antagônico na densidade da mosca branca, ou seja, quaisquer um dos cultivares testados parecer apresentar a mesma tolerância ao ataque deste inseto praga, independentemente das cultivares apresentarem ou não resistência a lagartas desfolhadoras.

Tabela 1: ANOVA de medidas repetidas para níveis de infestação de Bemisia tabaci em cultivares de soja Bt e não Bt. Os dados foram coletados semanalmenteentre 24 de maio a 26 de julho de 2017, em um total de nove avaliações, em um cultivo da soja no município de Montes Altos/MA.

\begin{tabular}{|c|c|c|c|c|c|c|c|c|c|}
\hline \multirow{2}{*}{$\begin{array}{l}\text { Fontes de } \\
\text { variação }\end{array}$} & \multicolumn{3}{|c|}{ adultos } & \multicolumn{2}{|c|}{ ovos } & \multicolumn{2}{|c|}{ ninfas } & \multicolumn{2}{|c|}{ total } \\
\hline & $\mathrm{df}$ & $\mathrm{F}$ & $P$ & $\mathrm{~F}$ & $P$ & $\mathrm{~F}$ & $P$ & $\mathrm{~F}$ & $P$ \\
\hline \multicolumn{10}{|c|}{ Entre níveis do fator } \\
\hline Cultivar (C) & 2 & 6,04 & $0.03 *$ & 3,36 & 0.09 & 1,49 & 0,29 & 5,23 & $0,04 *$ \\
\hline Bloco (B) & 4 & 2,57 & 0,13 & 0,70 & 0.62 & 6,22 & 0,02 & 1,54 & 0,29 \\
\hline Resíduo & 7 & - & - & - & - & - & - & & \\
\hline \multicolumn{10}{|c|}{ Dentro dos níveis do fator } \\
\hline Tempo (T) & 8 & 7,12 & $<0,001 * *$ & 15,53 & $<0,001 * * *$ & 7,81 & $<0,001 * * *$ & 13,24 & $<0,001 * * *$ \\
\hline $\mathrm{T} \times \mathrm{C}$ & 16 & 1,03 & 0,44 & 1,59 & 0,10 & 1,47 & 0,14 & 1,24 & 0,33 \\
\hline $\mathrm{T} \times \mathrm{B}$ & 32 & 1,18 & 0,28 & 0,75 & 0,80 & 0,69 & 0,87 & 0,78 & 0,64 \\
\hline Resíduo & 56 & - & & & & & & & \\
\hline
\end{tabular}

\section{Conclusões}

Dentre os cultivares de soja avaliados, não foi observada diferenças na densidade e flutuação populacional da mosca branca $B$. tabaci entre a cultivar não $\mathrm{Bt}$ e as cultivares $\mathrm{Bt}$ avaliadas. Portanto, as cultivares Bt resistentes a lagartas avaliadas podem ser utilizadas sem serem esperados efeitos sobre o inseto não alvo da tecnologia, neste caso, a mosca branca $B$. tabaci. 
A

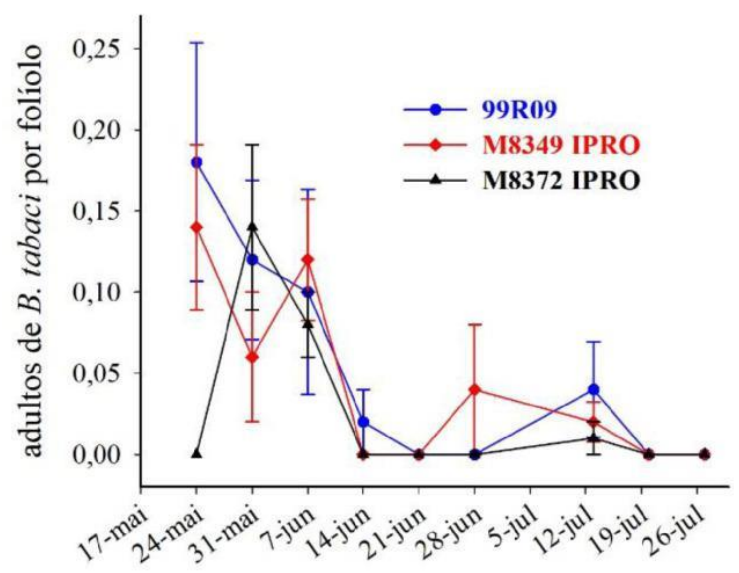

C

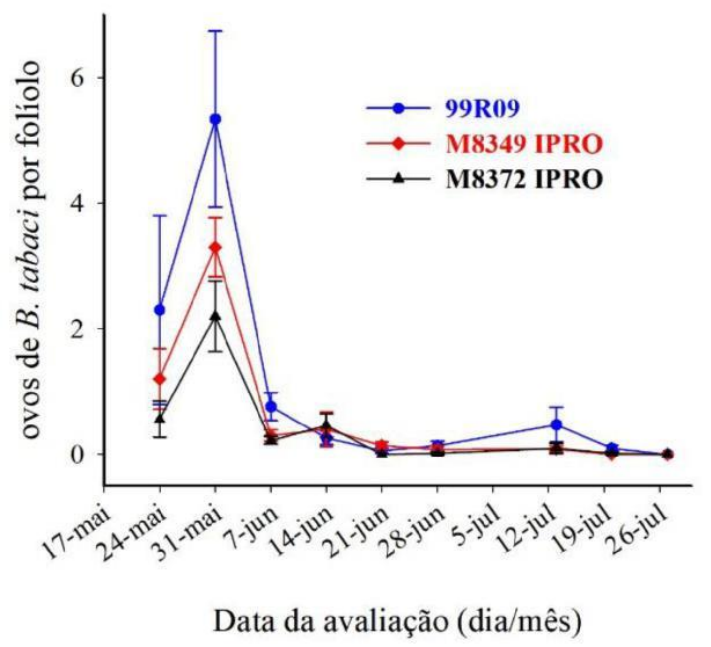

B

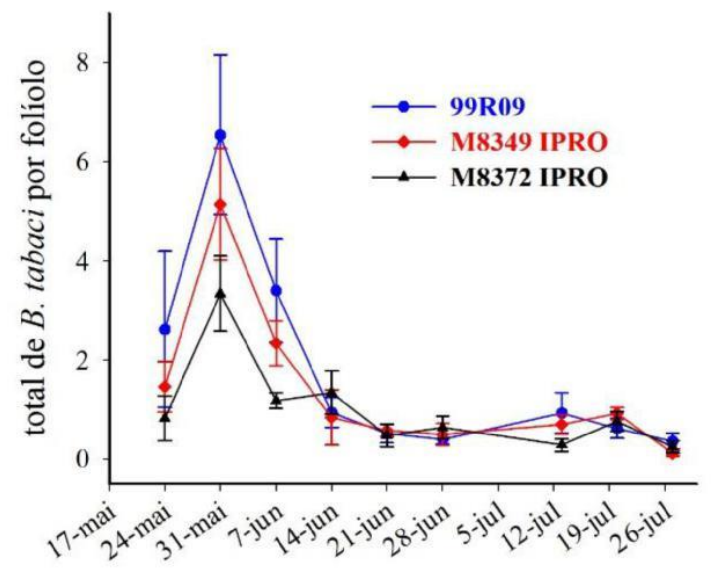

D

Data da avaliação (dia/mês)

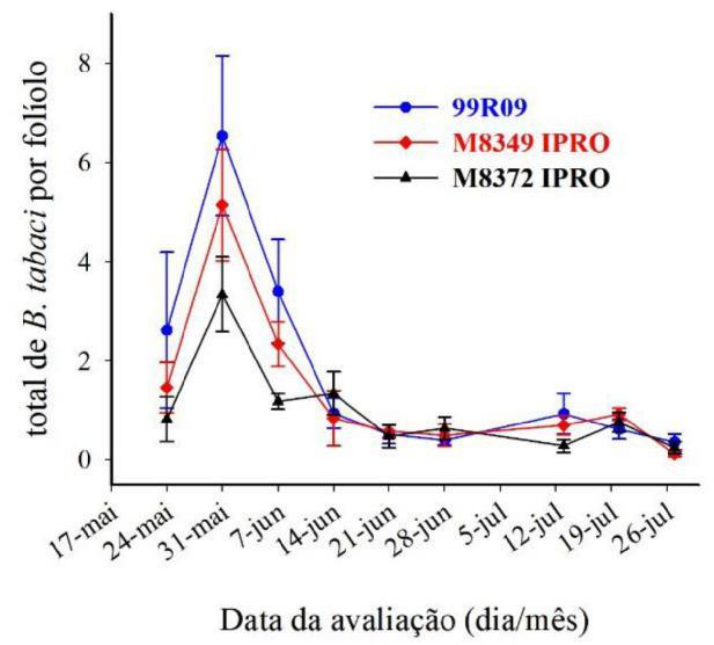

Figura 1. Densidade e flutuação populacional dos estádios de desenvolvimento de mosca branca, Bemisia tabaciem cultivares de soja Bt e não Bt:(A)adultos, (B)Número médio total; (C) ovos e (D) ninfas.

\section{Referências}

CHU, C.C.; FREEMAN, T.P.; BUCKNER, J.S.; HENNEBERRY, T.J.; NELSON, D.R.; NATWICK, E. Susceptibility of upland cotton cultivars to Bemisiatabaci biotype B (Homoptera: Aleyrodidae) in relation to leaf age and trichome density. Annals of Entomological Society of America, 94: 743-749, 2001.

CONAB. Boletim das safras. CONAB. 2017/2018. Disponível em<: https://www.conab.gov.br/info-agro/safras/graos/boletim-da-safra-de-graos. > Acessado em 11 setembro, 2018.

FOOD EN AGRICULTURE ORGANIZATION OF THE UNITED NATIONS-FAO. 2013. Disponível em: http://www.fao.org/brasil/pt/ Acesso em: 14 setembro, 2018. 
JAMES C. Global status of commercialized biotech/GM crops. ISAAA Brief No. 51. ISAAA, Ithaca, New York.2015.

LOPEZ V.; VOS, J.; POLAR, P.; KRAUSS, U. Discovery learning about sustainable management of whitefly pests and whitefly-borne viruses. International Center for Tropical Agriculture (CIAT), Cali, Colômbia, v. 1, n. 1, p. 12-37, 2008.

MAUAD, M.; et al.. Influência da densidade de semeadura sobre características agronômicas na cultura da soja. Revista Agrarian, v. 3, n. 9, p. 175-181, 2010.

MOSCARDI, F.; BUENO, A. F.; SOSA-GOMEZ, D. R.; ROGGIA, S.; HOFFMANN-CAMPO, C. B.; POMARI, A. F.; CORSO, I. C.; YANO, S. A. C. Artrópodes que atacam as folhas da soja.In: HOFFMANN-CAMPO, C. B.; CORREAA-FERREIRA, B. S.; MOSCARDI, F. (Ed.). Soja: manejo integrado de insetos e outros Artrópodes-praga. Brasília, DF: Embrapa, 2012.p. 213-334.

OLIVEIRA, C. E. S.; CARNEIRO, D. E. F.; TOSCANO, L. C.; FERREIRA, R. M. S.; ANDRADE, J. V. Preferência para oviposição de Bemisiatabaci(Gennadius 889) biótipo B em soja transgênica e não-transgênica. Tecnologia \& Ciência Agropecuária, João Pessoa-PB, v. 11, n. 6 , p. $7-10,2017$.

OLIVEIRA, C. E. S.; CARNEIRO, D. E. F.; TOSCANO, L. C.; SANTOS, R. M. F. Dinâmica populacional de Bemisiatabacibiótipo B (Gennadius, 1889) em cultivares de soja transgênica. Revista de Agricultura Neotropical, v. 5, n. 2, p. 1-5, 2018.

SAS INSTITUTE, 1989. SAS/STAT User's Guide, Version 6, SAS Institute, Cary. 